\title{
Oxygen Vacancies Nucleate Charged Domain Walls in Ferroelectrics
}

\author{
Petralanda, Urko; Kruse, Mads; Simons, Hugh; Olsen, Thomas
}

Published in:

Physical Review Letters

Link to article, DOI:

10.1103/PhysRevLett.127.117601

Publication date:

2021

Document Version

Publisher's PDF, also known as Version of record

Link back to DTU Orbit

Citation (APA):

Petralanda, U., Kruse, M., Simons, H., \& Olsen, T. (2021). Oxygen Vacancies Nucleate Charged Domain Walls in Ferroelectrics. Physical Review Letters, 127(11), [117601]. https://doi.org/10.1103/PhysRevLett.127.117601

\section{General rights}

Copyright and moral rights for the publications made accessible in the public portal are retained by the authors and/or other copyright owners and it is a condition of accessing publications that users recognise and abide by the legal requirements associated with these rights.

- Users may download and print one copy of any publication from the public portal for the purpose of private study or research.

- You may not further distribute the material or use it for any profit-making activity or commercial gain

- You may freely distribute the URL identifying the publication in the public portal 


\title{
Oxygen Vacancies Nucleate Charged Domain Walls in Ferroelectrics
}

\author{
Urko Petralanda $\odot,{ }^{1}$ Mads Kruse, ${ }^{1}$ Hugh Simons, ${ }^{2}$ and Thomas Olsen ${ }^{1, *}$ \\ ${ }^{1}$ Computational Atomic-Scale Materials Design (CAMD), Department of Physics, Technical University of Denmark, \\ 2800 Kgs. Lyngby, Denmark \\ ${ }^{2}$ Department of Physics, Technical University of Denmark, 2800 Kgs. Lyngby, Denmark
}

(Received 17 December 2020; revised 25 June 2021; accepted 12 August 2021; published 9 September 2021)

\begin{abstract}
We study the influence of oxygen vacancies on the formation of charged $180^{\circ}$ domain walls in ferroelectric $\mathrm{BaTiO}_{3}$ using first principles calculations. We show that it is favorable for vacancies to assemble in crystallographic planes, and that such clustering is accompanied by the formation of a charged domain wall. The domain wall has negative bound charge, which compensates the nominal positive charge of the vacancies and leads to a vanishing density of free charge at the wall. This is in contrast to the positively charged domain walls, which are nearly completely compensated by free charge from the bulk. The results thus explain the experimentally observed difference in electronic conductivity of the two types of domain walls, as well as the generic prevalence of charged domain walls in ferroelectrics. Moreover, the explicit demonstration of vacancy driven domain wall formation implies that specific charged domain wall configurations may be realized by bottom-up design for use in domain wall based information processing.
\end{abstract}

DOI: 10.1103/PhysRevLett.127.117601

Domain walls (DWs) in ferroelectric crystals are twodimensional topological defects separating domains of distinct directions of the spontaneous polarization. They are ubiquitous, and significantly affect physical properties [1-3] such as polarization switching [4,5], dielectric permittivity [6,7], and piezoeletric response [8,9]. In addition, DWs are typically highly mobile [10] and their position may be controlled by external electric fields. The versatile properties of DWs [11,12] have thus opened exciting avenues for applications in electronics, such as diodes [13] and nonvolatile memory devices [14,15], and are promising candidates as building blocks for the next generation of photovoltaics [16,17].

The properties of individual DWs strongly depend on the orientation of the spontaneous polarization $(\mathbf{P})$ with respect to the DW. When the normal component of $\mathbf{P}$ changes across the wall, a net bound charge is created in the DW [18], giving rise to electric fields that typically far exceed the coercive field for polarization reorientation. Such a charged domain wall (CDW) would be highly unstable without a mechanism to screen the bound charge [19-21]. As such, unambiguous verification that CDWs are present in proper ferroelectric crystals $[18,20,22,23]$ has led to the conclusion that charged impurities must play a fundamental role in stabilizing CDWs [24].

The role of oxygen vacancies $\left(V_{\mathrm{O}} \mathrm{s}\right)$ as a stabilizing agent for CDWs has been studied both experimentally and by simulations [25-31]. In addition, it is known that $V_{\mathrm{O}} \mathrm{s}$ may assemble in perovskite lattice planes under certain conditions [32-36]. However, these two effects have generally been regarded as unrelated, as the most widely accepted view is that $V_{\mathrm{O}}$ s serve to stabilize CDWs that have formed spontaneously, or by other means. However, previous studies have also shown that $V_{\mathrm{O}} \mathrm{s}$ located at axial sites of $\mathrm{TiO}_{6}$ octahedra in $\mathrm{PbTiO}_{3}$ create a displacement of the Ti atom [37] and a corresponding dipole moment. This suggests an alternative view in which vacancies may in fact facilitate the formation of CDWs instead of simply delivering a stabilizing charge distribution. The notion that $\mathrm{V}_{\mathrm{O}} \mathrm{S}$ may directly nucleate CDWs has broad implications for the understanding and application of CDWs in general. However, the formation mechanisms of CDWs remain elusive due to the apparent strong instability of CDWs and the technical challenges associated with carrying out $a b$ initio studies of CDWs in both pristine and doped ferroelectrics.

In this Letter, we describe results from first-principles calculations of $180^{\circ}$ domain walls in $\mathrm{BaTiO}_{3}$ showing that it is favorable for oxygen vacancies to accumulate in planes, and that such accumulation gives rise to CDWs forming spontaneously. In particular, we demonstrate that the screening of the bound charge of head-to-head $(\mathrm{HH})$ CDWs occurs through the filling of local conduction bands, regardless of vacancies. The negative charge at tail-to-tail (TT) CDWs is screened by the positive charge from the vacancies, thus quenching the $p$-type conductivity characterizing TT CDWs in the pristine system.

$\mathrm{BaTiO}_{3}$ presents a phase transition from cubic $(P m \overline{3} m)$ to tetragonal $(P 4 \mathrm{~mm})$ structure at $\sim 404 \mathrm{~K}$, a subsequent first-order transition to an orthorhombic (Amm2) structure at $\sim 273 \mathrm{~K}$ and finally another first-order transition at $\sim 183 \mathrm{~K}$ to a rhombohedral $(R 3 \mathrm{~m})$ structure with polarization in the (111) direction [38,39]. Here we will focus on the tetragonal phase, but expect that the conclusions will hold true for other phases and similar compounds. 
Our calculations were done in the framework of density functional theory (DFT), implemented in the GPAW electronic structure package $[40,41]$ using the projectoraugmented wave method [42], the local density approximation (LDA) [43] and a plane wave basis. We used a plane-wave cutoff of $700 \mathrm{eV}$ and a $\Gamma$-centered MonkhorstPack $k$-point grid with a density of $6 \AA$. Forces were typically relaxed below $0.01 \mathrm{eV} / \AA$ (for details on the atomic structure and Born effective charges see the Supplemental Material [44]).

We begin by considering $180^{\circ} \mathrm{CDWs}$ in $\mathrm{BaTiO}_{3}$ without any vacancies. This will serve as a reference system that allows us to gain insight into the influence of vacancies on the electronic properties of CDWs. In addition, it is known that a dilute distribution of CDWs in oxide perovskites can be stable and robust in defect-free thin films [22]. We thus construct a $1 \times 1 \times 16$ supercell of tetragonal $\mathrm{BaTiO}_{3}$ and divide it into two areas of opposite polarization parallel to the long axis of the supercell. The polarization of the subcell $\alpha$ can be written as $P_{i, \alpha}=(1 / \Omega) \sum_{j, a \in \alpha} Z_{i j}^{* a} d_{j}^{a}$ [47]. Here $\Omega$ is the unit cell volume, $d_{j}^{a}$ is the displacement of atom $a$ with respect to its position in the centrosymmetric structure in direction $j, Z_{i j}^{* a}$ is the Born effective charge tensor of atom $a$ and the sum runs over atoms in unit cell $\alpha$. Since the $Z_{i j}^{* a}$ depend on the local electronic structure, we average the tensors obtained for the cubic and tetragonal phases of $\mathrm{BaTiO}_{3}$ [44]. The atomic displacements of the supercell are smoothed so the polarization profile becomes $P_{\alpha}=P_{0} \tanh \left(z_{\alpha} / \delta\right)$, where $z_{\alpha}$ is the center position of unit cell $\alpha$ and $P_{0}$ is the magnitude of the calculated bulk polarization, $0.24 \mathrm{C} / \mathrm{m}^{2}$, which is in good agreement with the experimental value of $0.26 \mathrm{C} / \mathrm{m}^{2}$ $[48,49]$ and computational works using the LDA [50,51]. We set $\delta=1.75$ as the structural width of the wall and adopt $\mathrm{BaO}$ centered DWs, since the $V_{\mathrm{O}}$ s used in the simulations described below are more stable at these planes. We did not relax the structure, as it would so be driven into a single domain. Finally, we emphasize that, although this structure is a somewhat artificial representation of a CDW, its primary intention is to unravel the basic principles of screening in the system.

Figure 1 shows a schematic representation of the supercell, including the profile of polarization per unit cell as calculated following the procedure in Ref. [47]. We also show the bound charge density $\rho_{b}$ arising from the polarization profile, as well as the electrostatic potential obtained from DFT. As expected, a positive (negative) bound charge density peak is located at the HH (TT) wall and is accompanied by a minimum (maximum) of the potential. The integrated bound charge density at each of the walls has magnitude $2 P_{0}$ by construction and the potential energy difference $(\Delta V)$ between the walls is roughly $2.3 \mathrm{eV}$. Since the potential between the walls is linear, the electric fields inside the two domains can be

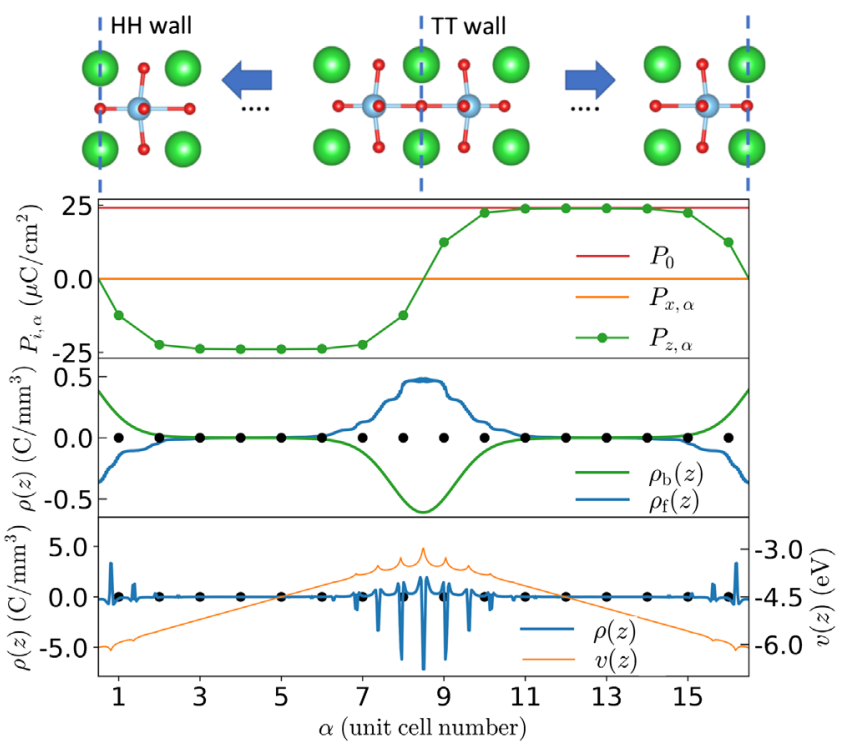

FIG. 1. Properties of the unrelaxed CDWs in a $1 \times 1 \times 16$ supercell. Top: schematic atomic structure with exagerated displacements. The blue arrows indicate the direction of polarization; green, blue and red spheres indicate $\mathrm{Ba}, \mathrm{Ti}$, and $\mathrm{O}$ atoms, respectively. The dots indicate positions of $\mathrm{Ti}$ atoms in the individual unit cells. Second from top: polarization profile of the supercell. Second from bottom: bound $\rho_{b}$ and smoothed free $\rho_{\mathrm{f}}$ charge density. Bottom: electrostatic potential energy $v(z)$ averaged over the plane orthogonal to $z$ and total charge density $\rho$ obtained from a sliding window average.

regarded as constant and the total charge density at the CDWs are then related to the potential energy difference by Gauss law as

$$
\sigma_{\mathrm{tot}}(d)=\frac{\varepsilon_{0} \Delta V / e}{d}
$$

where $d$ is the distance between the walls and $\varepsilon_{0}$ is the vacuum permittivity. Inserting the values obtained from DFT yields a charge density of $\sigma_{\text {tot }}=0.0048 \mathrm{C} / \mathrm{m}^{2}$. This is 2 orders of magnitude smaller than the bound charge indicating that the bound charge is almost fully compensated by free charge.

The mechanism behind the screening can be envisioned by considering two CDWs in close proximity with bound charge densities $\pm 2 P_{0}$. Without any screening mechanism, the electric field between the walls would be determined by the polarization $P_{0}$ only. However, if the distance between the walls is increased, the potential difference between the walls increases (due to the constant electric field) and the conduction (valence) bands are lowered (raised) at the $\mathrm{HH}$ (TT) until they are aligned [22,23,52]. At this point, charge will be transferred between the walls to align the Fermi levels at the walls, and the potential energy difference will be pinned at the value of the band gap. Thus, when the difference between the walls $d$ is increased in Eq. (1), $\Delta V$ 
will remain fixed while the electric field and charge density at the walls decrease.

For CDWs at large separation, the charge density will thus be finite, albeit orders of magnitude smaller than the bound charge. In our calculations we obtain a LDA band gap for $\mathrm{BaTiO}_{3}$ of $1.9 \mathrm{eV}$, which is in agreement with the potential difference of $2.3 \mathrm{eV}$. Note that for such a "pristine" CDW structure, the total charge density at the walls is roughly determined by the band gap and the distance between the walls, whenever $d$ exceeds the "short-circuit distance" $d_{\mathrm{sc}}=\varepsilon_{0} E_{\mathrm{gap}} / 2 e P_{0}$. Integrating the charge density through the CDW (obtained from a sliding window average (see Supplemental Material [44] for details) yields $0.005 \mathrm{C} / \mathrm{m}^{2}$, which is in agreement with the result obtained from Eq. (1). We have checked that the calculated potential difference is the same for $1 \times 1 \times 8$ and $1 \times 1 \times 12$ unit cell systems.

The screening described above is more clearly visualized from the band structure and projected density of states (PDOS) resolved in individual unit cells, shown in Fig. 2. Given the indirect band gap in $\mathrm{BaTiO}_{3}$, the electron (hole) doping at the $\mathrm{HH}$ (TT) walls occurs at different locations in the $2 \mathrm{D}$ Brillouin zone. The band structure shows that the $\Gamma$ point mediates the electron doping at the $\mathrm{HH}$ wall, whereas the hole doping mainly occurs at the $M$ point. Resolving the PDOS in contributions from different unit cells yields a profile reminiscent of the electrostatic potential. For a given unit cell, the PDOS resembles that of bulk $\mathrm{BaTiO}_{3}$, but shifted according to the local value of the electrostatic potential. The PDOS also implies that charge carriers in the vicinity of the Fermi level are strictly localized at the two CDWs as expected, which implies that we can calculate the free charge density at the HH (TT) wall by adding the norm-squared wave functions of the conductive states below(above) the Fermi level (see Supplemental Material for details [44]). This procedure yields a free charge density of $\pm 0.40 \mathrm{C} / \mathrm{m}^{2}$ at the two walls, which (almost) cancel the bound charge density at the two walls as anticipated. The smoothed free charge density is shown in Fig. 1 and exhibits a profile that compensates the bound charge.
In order to study the role of $V_{\mathrm{O}} \mathrm{S}$ in the formation of CDWs, we use a bulk, single domain represented in a $3 \times$ $3 \times 8$ supercell as the starting point. We find the most stable position for a planar distribution of oxygen vacancies is the $\mathrm{BaO}$ plane (see Fig. S2 in the Supplemental Material [44]), although the formation energies of a single vacancy are similar in both planes [53]. We therefore begin by introducing one vacancy into a $\mathrm{BaO}$ plane, and fully relax the supercell (see the top part of Fig. 3). Following this relaxation, we see that the vacancy strongly repels the neighboring Ti atom towards the opposite direction of the initial polarization, thereby decreasing the local polarization, as shown in Fig. 3. This occurs through the breaking of the bonding orbital formed by the emptied $\mathrm{O} p$ and the Ti $t_{2 g}$, in a similar scenario to that described by Park et al. for $\mathrm{PbTiO}_{3}$ [37]. We note that we obtain the same configuration if we set an artificial DW in a $3 \times 3 \times 8$ supercell, put a vacancy on it and relax it.

We will now show that it is favorable for additional vacancies to migrate to a plane with an initial amount of vacancies. To this aim, we define the energy cost of adding the $i$ th vacancy in unit cell $\alpha$ relative to the energy cost of adding a single vacancy in a bulk $\mathrm{BaO}$ plane:

$$
\Delta E_{i \alpha}=E_{i \alpha}-E_{(i-1) \alpha_{0}}-\left(E_{1}-E_{\text {bulk }}\right), \quad i>1,
$$

where $E_{\text {bulk }}$ is the energy of a single domain without vacancies, $E_{1}$ is the energy of the supercell with a single vacancy in any $\mathrm{BaO}$ plane, and $E_{i \alpha}$ is the energy of the supercell with the $i$ th vacancy placed in unit cell $\alpha$ and $i-1$ vacancies at their optimal positions $\left(\alpha_{0}\right)$.

We first calculate $E_{1 \alpha}$, starting with a fully relaxed configuration with a vacancy in the $\mathrm{BaO}$ plane, and calculate the energy of the structure with a second vacancy in all the possible $\mathrm{BaO}$ planes. The result, shown in Fig. 3, shows that the optimal position of the second vacancy is the plane where the first vacancy was placed. We have checked that vacancies in the $\mathrm{TiO}_{2}$ plane are always more unfavorable (see Fig S3 in the Supplemental Material [44]). We

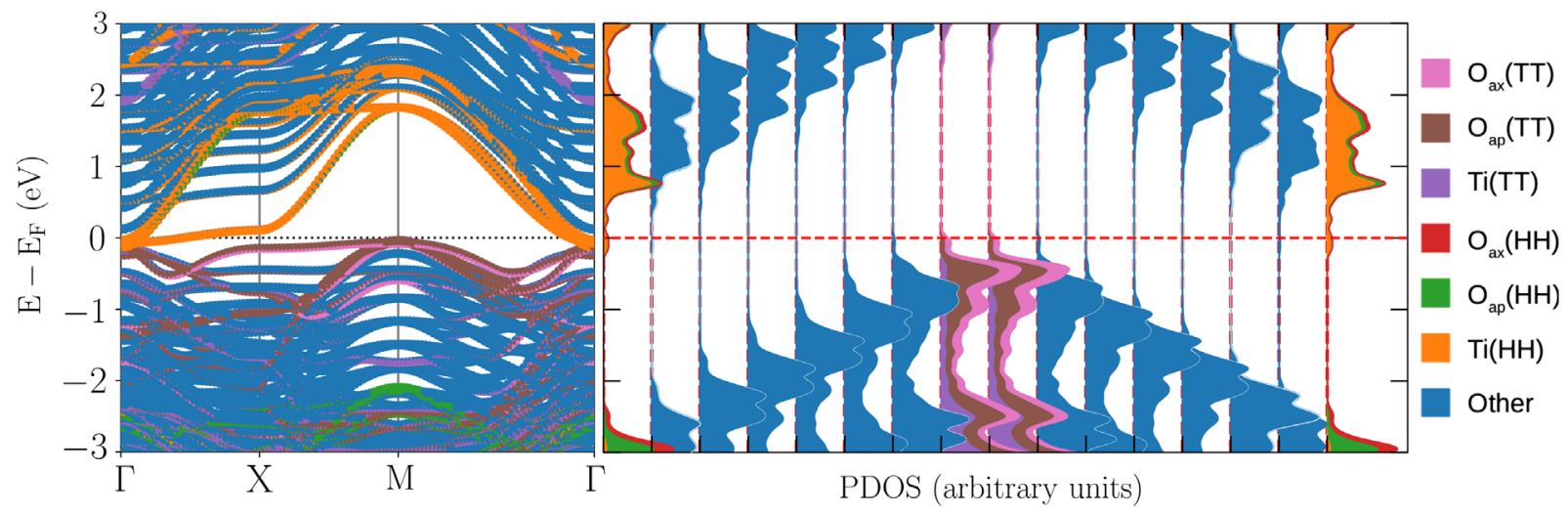

FIG. 2. Left: projected band structure of the unrelaxed $1 \times 1 \times 16$ supercell with no vacancies. Right, the projected density of states resolved in individual unit cells. 

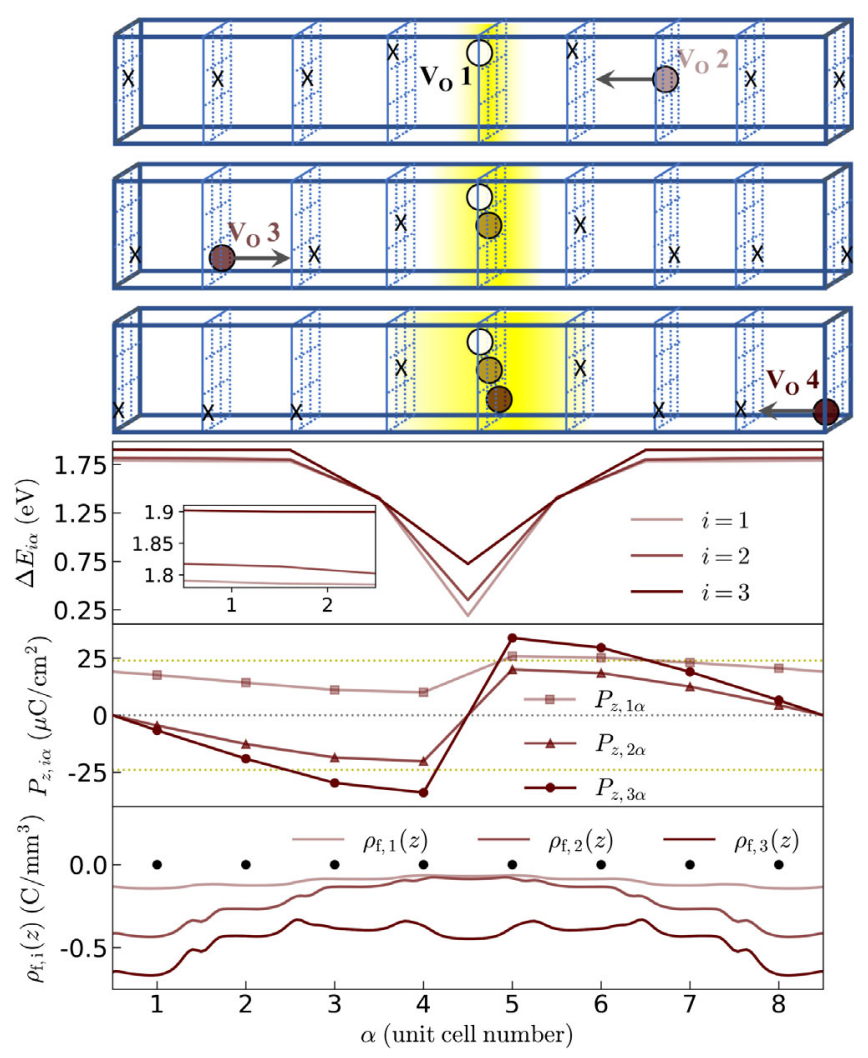

FIG. 3. Top: Schematic illustration of the vacancy migration that leads to domain wall formation in a $3 \times 3 \times 8$ supercell. The crosses indicate the lowest formation energy positions at each $\mathrm{BaO}$ plane. Below, we show the energy cost $\Delta E_{i \alpha}$ for the $i$ th vacancy at position $\alpha$ (see text) given that $i-1$ vacancies are situated at their optimal positions. $P_{z, i \alpha}$ is the polarization profile of the supercell with $i$ vacancies at their optimal positions. The bottom panel shows the smoothed free $\left(\rho_{f}\right)$ charge density profiles for the relaxed supercell with one, two, and three vacancies at the central $\mathrm{BaO}$ plane.

then calculate $E_{2 \alpha}$ by the same procedure starting with the relaxed structure with two vacancies in a single $\mathrm{BaO}$ plane. Again, the optimal position is the $\mathrm{BaO}$ plane where the other vacancies are located. And the same happens to the fourth vacancy, although we see that it experiences a weaker attraction to the three initial vacancies [see Fig. 3], indicating that once enough vacancies needed to form the wall are assembled, the attraction for further vacancies decreases. This attraction between vacancies may seem counterintuitive, since they present a charge of $+2 e$ and should thus repel one another. However, the vacancies induce a polarization that initiates a negatively charged DW, which in turn provides the attractive long-ranged force between the vacancies. This process is evident in Fig. 3, where we show the polarization profiles for 1,2 , and 3 vacancies at their optimal positions in the third panel, starting from the top. The first vacancy introduces a local polarization reminiscent of a negatively charged domain wall, attracting the next vacancy. When additional vacancies diffuse to the optimal position at the wall the local polarization is distorted further, until a TT DW is fully formed. This suggests that accumulation of oxygen vacancies in planes occurs naturally in the material. Depending on the initial distribution of vacancies in the crystal, they could potentially accumulate in alternative plane geometries creating other types of DWs such as 90 degree walls [54].

In our simulations a single vacancy corresponds to a $11 \%$ oxygen depletion in a single $\mathrm{BaO}$ plane. It is natural to ask whether our findings will hold true at realistic vacancy distributions. For a random distribution of vacancies there will inevitably be some planes with a higher concentration than others, which will act as sinks for vacancies of the neighboring environment. The actual accumulation will be mediated through thermally assisted diffusion, however, a quantitative estimation of diffusion rates is beyond the scope of this work.

It is instructive to compare the smoothed free charge density profiles shown in Fig. 3 during the DW formation process, with the case without vacancies shown in Fig. 1. In all cases we see a similar picture at the $\mathrm{HH}$ wall, where the negative free charge screens the positive bound charge. In contrast, as the TT wall is formed it presents nearly no free charge since the bound charge already compensates the positive charge of the $V_{\mathrm{O}} \mathrm{s}$. In fact, the apparent finite charge at the TT wall in Fig. 3 is nearly exclusively an artifact of the finite supercell size as shown in the Supplemental Material [44], where we also show the raw charge densities used to calculate smoothed free charge profiles in Fig. 3. Once seeded, the HH-TT DW pair are structurally protected topological defects. As shown in Fig. 1, the HH wall is not fully screened by free charge and this enforces a net negative charge in the TT wall area. Because of charge neutrality we have $\sigma_{f}+2 e n_{\mathrm{vac}}=0$, where $\sigma_{f}$ is the total free charge in the supercell and $n_{\text {vac }}$ the vacancy number at the wall. This is verified in the increase of free charge at the TT wall at increasing $n_{\text {vac }}$, as shown in Fig. 3 and Table S3 in the Supplemental Material [44]. In addition the complete screening for well separated walls implies $\sigma_{f, \mathrm{HH}} \sim \sigma_{b, \mathrm{HH}}$ and we conclude that the optimal vacancy density at the wall, when $\sigma_{f, \mathrm{TT}}=0$, is given by $n_{\text {vac, } 0} \sim\left(P_{0} / e\right)$. In our system this number lies between two and three vacancies per supercell. Figure 3 suggests the concentration of DW vacancies saturates once the wall is formed. As additional vacancies provide a localized screening charge for the TT bound charge, the ability of the TT wall to conduct is very limited, in agreement with experiments [24]. Moreover, such extra free charge will be manifestly delocalized, unlike the pristine wall free charge (see Fig. S4 in the Supplemental Material [44]), reducing the potential difference between walls (see Fig. S5 [44]) and significantly increasing their stability. Compared to the pristine case, the conductivity mechanism of the $\mathrm{HH}$ wall remains essentially unaffected though; Ti $t_{2 g}$ states from the 
conduction band shift below the Fermi level in the HH wall area, similarly to the pristine system (see Figs. S1 and S6 in Ref. [44]).

In conclusion, we have shown that, having an initial oxygen vacancy cluster in tetragonal $\mathrm{BaTiO}_{3}$, it is favorable for other vacancies to migrate to the $\mathrm{BaO}$ plane defined by this cluster and that a TT domain wall is formed in the process. The driving force is the negative bound charge emerging at the TT wall. It naturally follows from our calculations that oxygen vacancies are attracted to TT DWs, which has already been suggested in the past $[24,32]$. However, the fact that oxygen vacancies play a critical role in the formation of CDWs has not been demonstrated previously and provides a significant indication as to why and how CDWs form. Moreover, the implications of this mechanism are potentially far reaching, since it suggests that particular CDW distributions may be accomplished by simply seeding a ferroelectric with a suitable distribution of vacancies. Such control will be a crucial ingredient for the future development of domain wall nanoelectronics.

T. O. and U.P. were supported by the Villum Foundation, Grant No. 00028145.

*tolsen@fysik.dtu.dk

[1] G. Catalan, J. Seidel, R. Ramesh, and J. F. Scott, Domain wall nanoelectronics, Rev. Mod. Phys. 84, 119 (2012).

[2] P. S. Bednyakov, B. I. Sturman, T. Sluka, Alexander K. Tagantsev, and Petr V. Yudin, Physics and applications of charged domain walls, Comput. Mater. Sci. 4, 65 (2018).

[3] G. F. Nataf, M. Guennou, J. M. Gregg, D. Meier, J. Hlinka, E. K. H. Salje, and J. Kreisel, Domain-wall engineering and topological defects in ferroelectric and ferroelastic materials, Nat. Rev. Phys. 2, 634 (2020).

[4] S. Liu, I. Grinberg, and A. Rappe, Intrinsic ferroelectric switching from first principles, Nature (London) 534, 360 (2016).

[5] L. Li, J. Britson, J. R. Jokisaari, Y. Zhang, C. Adamo, A. Melville, D. G. Schlom, L.-Q. Chen, and X. Pan, Giant resistive switching via control of ferroelectric charged domain walls, Adv. Mater. 28, 6574 (2016).

[6] J. Fousek and V. Janoušek, The contribution of domain-wall oscillations to the small-signal permittivity of triglycine sulphate, Phys. Status Solidi B 13, 195 (1966).

[7] Pavlo Zubko, J. C. Wojdeł, M. Hadjimichael, S. FernandezPena, A. Sené, I. Luk'yanchuk, J.-M. Triscone, and J. Íniguez, Negative capacitance in multidomain ferroelectric superlattices, Nature (London) 534, 524 (2016).

[8] G. Arlt and H. Dederichs, Complex elastic, dielectric and piezoelectric constants by domain wall damping in ferroelectric ceramics, Ferroelectrics 29, 47 (1980).

[9] T. Rojac, H. Ursic, A. Bencan, B. Malic, and D. Damjanovic, Mobile domain walls as a bridge between nanoscale conductivity and macroscopic electromechanical response, Adv. Funct. Mater. 25, 2099 (2015).

[10] T. Tybell, P. Paruch, T. Giamarchi, and J.-M. Triscone, Domain Wall Creep in Epitaxial Ferroelectric
$\mathrm{Pb}\left(\mathrm{Zr}_{0.2} \mathrm{Ti}_{0.8}\right) \mathrm{O}_{3}$ Thin Films, Phys. Rev. Lett. 89, 097601 (2002).

[11] S. Y. Yang, J. Seidel, S. J. Byrnes, P. Shafer, C.-H. Yang, M. D. Rossell, P. Yu, Y.-H. Chu, J. F. Scott, J. W. Ager, L. W. Martin, and R. Ramesh, Above-bandgap voltages from ferroelectric photovoltaic devices, Nat. Nanotechnol. 5, 143 (2010).

[12] X. Wu, U. Petralanda, L. Zheng, Y. Ren, R. Hu, S.-W. Cheong, S. Artyukhin, and K. Lai, Low-energy structural dynamics of ferroelectric domain walls in hexagonal rare-earth manganites, Sci. Adv. 3, e1602371 (2017)

[13] J. Whyte and J. A. Gregg, A diode for ferroelectric domainwall motion, Nat. Commun. 6, 7361 (2015).

[14] P. Sharma, Q. Zhang, D. Sando, C. Hou Lei, Y. Liu, J. Li, V. Nagarajan, and J. Seidel, Nonvolatile ferroelectric domain wall memory, Sci. Adv. 3, e1700512 (2017).

[15] J. Jiang, Z. Long Bai, Z. Hui Chen, L. He, D. Wei Zhang, Q. Hua Zhang, J. An Shi, M. Hyuk Park, J. F. Scott, C. Seong Hwang, and A. Quan Jiang, Temporary formation of highly conducting domain walls for non-destructive read-out of ferroelectric domain-wall resistance switching memories, Nat. Mater. 17, 49 (2018).

[16] A. M. Cook, B. M. Fregoso, F. de Juan, S. Coh, and J. E. Moore, Design principles for shift current photovoltaics, Nat. Commun. 8, 14176 (2017).

[17] A. Zenkevich, Yu. Matveyev, K. Maksimova, R. Gaynutdinov, A. Tolstikhina, and V. Fridkin, Giant bulk photovoltaic effect in thin ferroelectric $\mathrm{BaTiO}_{3}$ films, Phys. Rev. B 90, 161409(R) (2014).

[18] J. Seidel, L. W. Martin, Q. He, Q. Zhan, Y.-H. Chu, A. Rother, M. E. Hawkridge, P. Maksymovych, P. Yu, M. Gajek, N. Balke, S. V. Kalinin, S. Gemming, F. Wang, G. Catalan, J. F. Scott, N. A. Spaldin, J. Orenstein, and R. Ramesh, Conduction at domain walls in oxide multiferroics, Nat. Mater. 8, 229 (2009).

[19] B. M. Vul, G. M. Guro, and I. I. Ivanchik, Encountering domains in ferroelectrics, Ferroelectrics 6, 29 (1973).

[20] T. Sluka, A. K. Tagantsev, P. Bednyakov, and N. Setter, Free-electron gas at charged domain walls in insulating $\mathrm{BaTiO}_{3}$, Nat. Commun. 4, 1808 (2013).

[21] D. R. Småbråten, Q. N. Meier, S. H. Skjærvø, K. Inzani, D. Meier, and S.M. Selbach, Charged domain walls in improper ferroelectric hexagonal manganites and gallates, Phys. Rev. Mater. 2, 114405 (2018).

[22] M. Y. Gureev, A. K. Tagantsev, and N. Setter, Head-to-head and tail-to-tail $180^{\circ}$ domain walls in an isolated ferroelectric, Phys. Rev. B 83, 184104 (2011).

[23] J. Sifuna, P. García-Fernández, G. S. Manyali, G. Amolo, and J. Junquera, First-principles study of two-dimensional electron and hole gases at the head-to-head and tail-to-tail $180^{\circ}$ domain walls in $\mathrm{PbTiO}_{3}$ ferroelectric thin films, Phys. Rev. B 101, 174114 (2020).

[24] P. S. Bednyakov, T. Sluka, A. K. Tagantsev, D. Damjanovic, and N. Setter, Formation of charged ferroelectric domain walls with controlled periodicity, Sci. Rep. 5, 15819 (2015).

[25] L. He and D. Vanderbilt, First-principles study of oxygenvacancy pinning of domain walls in $\mathrm{PbTiO}_{3}$, Phys. Rev. $\mathrm{B}$ 68, 134103 (2003). 
[26] V. Gopalan, V. Dierolf, and D. A. Scrymgeour, Defectdomain wall interactions in trigonal ferroelectrics, Annu. Rev. Mater. Res. 37, 449 (2007).

[27] D. Shilo, G. Ravichandran, and K. Bhattacharya, Investigation of twin-wall structure at the nanometre scale using atomic force microscopy, Nat. Mater. 3, 453 (2004).

[28] W. T. Lee, E. K. H. Salje, and U. Bismayer, Influence of point defects on the distribution of twin wall widths, Phys. Rev. B 72, 104116 (2005).

[29] J. J. Gong, C. F. Li, Y. Zhang, Y. Q. Li, S. H. Zheng, K. L. Yang, R. S. Huang, L. Lin, Z. B. Yan, and J.-M. Liu, Interactions of charged domain walls and oxygen vacancies in $\mathrm{BaTiO}_{3}$ : A first-principles study, Mater. Today Phys. 6, 9 (2018).

[30] W. R. Geng, X. H. Tian, Y. X. Jiang, Y. L. Zhu, Y. L. Tang, Y. J. Wang, M. J. Zou, Y. P. Feng, B. Wu, W. T. Hu, and $\mathrm{X}$. L. Ma, Unveiling the pinning behavior of charged domain walls in $\mathrm{BiFeO}_{3}$ thin films via vacancy defects, Acta Mater. 186, 68 (2020).

[31] Y.-M. Kim, A. Morozovska, E. Eliseev, M. P. Oxley, R. Mishra, S. M. Selbach, T. Grande, S. T. Pantelides, S. V. Kalinin, and A. Y. Borisevich, Direct observation of ferroelectric field effect and vacancy-controlled screening at the $\mathrm{BiFeO}_{3} / \mathrm{La}_{x} \mathrm{Sr}_{1-x} \mathrm{MnO}_{3}$ interface, Nat. Mater. 13, 1019 (2014).

[32] J. F. Scott and M. Dawber, Oxygen-vacancy ordering as a fatigue mechanism in perovskite ferroelectrics, Appl. Phys. Lett. 76, 3801 (2000).

[33] Y. Kim, A. S. Disa, T. E. Babakol, X. Fang, and J. D. Brock, Strain and oxygen vacancy ordering in $\mathrm{srtio}_{3}$ : Diffuse X-ray scattering studies, Phys. Rev. B 92, 064105 (2015).

[34] M. D. Glinchuk, E. A. Eliseev, G. Li, J. Zeng, S. V. Kalinin, and A. N. Morozovska, Ferroelectricity induced by oxygen vacancies in relaxors with perovskite structure, Phys. Rev. B 98, 094102 (2018).

[35] D. I. Woodward, I. M. Reaney, G. Y. Yang, E. C. Dickey, and C. A. Randall, Vacancy ordering in reduced barium titanate, Appl. Phys. Lett. 84, 4650 (2004).

[36] G. Deng, G. Li, A. Ding, and Q. Yin, Evidence for oxygen vacancy inducing spontaneous normal-relaxor transition in complex perovskite ferroelectrics, Appl. Phys. Lett. 87, 192905 (2005).

[37] C. H. Park and D. J. Chadi, Microscopic study of oxygenvacancy defects in ferroelectric perovskites, Phys. Rev. B 57, R13961 (1998).

[38] B. Ravel, E. A. Stern, R. I. Vedrinskii, and V. Kraizman, Local structure and the phase transitions of $\mathrm{BaTiO}_{3}$, Ferroelectrics 206, 407 (1998).

[39] J. Hlinka and P. Márton, Phenomenological model of a 90 domain wall in $\mathrm{BaTiO}_{3}$-type ferroelectrics, Phys. Rev. B 74, 104104 (2006).

[40] J. Enkovaara et al., Electronic structure calculations with GPAW: A real-space implementation of the projector augmented-wave method, J. Phys. Condens. Matter 22, 253202 (2010).

[41] A. Hjorth Larsen et al., The atomic simulation environment -a python library for working with atoms, J. Phys. Condens. Matter 29, 273002 (2017).

[42] P. E. Blöchl, Projector augmented-wave method, Phys. Rev. B 50, 17953 (1994).

[43] J. P. Perdew and Y. Wang, Accurate and simple analytic representation of the electron-gas correlation energy, Phys. Rev. B 45, 13244 (1992).

[44] See Supplemental Material at http://link.aps.org/ supplemental/10.1103/PhysRevLett.127.117601 for additional details of the bulk structure and Born effective charges of $\mathrm{BaTiO}_{3}$, the free and total charge profile calculation methods, the determination of the crystallographic planes for vacancies, the free charge profiles along the domain wall formation process, and density of states plots, which includes Refs. [45,46].

[45] P. Ghosez, X. Gonze, P. Lambin, and J.-P. Michenaud, Born effective charges of barium titanate: Band-by-band decomposition and sensitivity to structural features, Phys. Rev. B 51, 6765 (1995).

[46] M. Stengel, P. Aguado-Puente, N. A. Spaldin, and J. Junquera, Band alignment at metal/ferroelectric interfaces: Insights and artifacts from first principles, Phys. Rev. B 83, 235112 (2011).

[47] B. Meyer and David Vanderbilt, Ab initio study of ferroelectric domain walls in $\mathrm{PbTiO}_{3}$, Phys. Rev. B 65, 104111 (2002).

[48] Landolt-Bornstein Numerical Data and Functional Relationships in Science and Technology, Group III, edited by T. Mitsui (Springer, Berlin, 1981), Vol. 16.

[49] Landolt-Bornstein Numerical Data and Functional Relationships in Science and Technology, Group III, edited by E. Nakamura (Springer, Berlin, 1981), Vol. 28.

[50] W. Zhong, David Vanderbilt, and K. M. Rabe, Firstprinciples theory of ferroelectric phase transitions for perovskites: The case of $\mathrm{BaTiO}_{3}$, Phys. Rev. B 52, 6301 (1995).

[51] Y. Iwazaki, T. Sakashita, T. Suzuki, Y. Mizuno, and S. Tsuneyuki, First-principles calculations of structural changes induced by oxygen vacancies in tetragonal phase $\mathrm{BaTiO}_{3}$, Key Eng. Mater. 485, 19 (2011).

[52] K. Rahmanizadeh, D. Wortmann, G. Bihlmayer, and S. Blugel, Charge and orbital order at head-to-head domain walls in $\mathrm{PbTiO}_{3}$, Phys. Rev. B 90, 115104 (2014).

[53] L. Bainian, Y. Ma, Y. Zhou, and J. Ding, Single vacancies and vacancy pairs in tetragonal $\mathrm{BaTiO}_{3}$ : A first principles study, Ferroelectrics 401, 36 (2010).

[54] X. Wang, T. Xu, F. Xuan, C.-Q. Chen, T. Shimada, and T. Kitamura, Effect of the oxygen vacancy on the ferroelectricity of $90^{\circ}$ domain wall structure in $\mathrm{PbTiO}_{3}$ : A density functional theory study, J. Appl. Phys. 126, 174107 (2019). 